\title{
SOME THEOREMS ON EQUICONNECTED AND LOCALLY EQUICONNECTED SPACES
}

\author{
BY \\ CHARLES J. HIMMELBERG $\left({ }^{1}\right)$
}

1. Introduction. In the theory of retracts for finite-dimensional metrizable spaces it is well known that the absolute retracts are the spaces which are both contractible and locally contractible and that the absolute neighborhood retracts are the spaces which are locally contractible (Kuratowski [ 10, p. 289] and Dugundji [4, p. 244]). Thus it seems natural to drop the finite-dimensionality condition and try to describe absolute retracts and absolute neighborhood retracts in terms of some strong kind of contractibility. One is quickly led to consider the concept of local equiconnectedness (introduced by Fox in [5] and called ULC by Serre in $[12$, p. 490$])$. In the first part of this paper we discuss these concepts and some results relating them to the theory of retracts. Having done this we prove some theorems on the union of (locally) equiconnected spaces analogous to well-known theorems for absolute (neighborhood) retracts.

2. Equiconnected spaces and absolute retracts. In this paper an absolute retract $(\mathrm{AR})$ is a metrizable space which is a retract of each metrizable space containing it as a closed subspace. An absolute neighborhood retract (ANR) is a metrizable space which is a neighborhood retract of each metrizable space containing it as a closed subspace.

Let $X$ be a topological space, let $A$ be a subset of the product space $X \times X$ and let $\tau$ be a map (= continuous function) from $A \times I$ (where $I$ is the unit interval) to $X . \tau$ is said to have the connecting property on $A$ iff $\tau(x, y, 0)=x, \tau(x, y, 1)=y$, for all $(x, y) \in A$, and $\tau(x, x, I)=x$ for any $(x, x) \in A$. A topological space $X$ is equiconnected (EC) iff there is a map $\tau$ from $X \times X \times I$ to $X$ which has the connecting property on $X \times X . X$ is locally equiconnected (LEC) iff there is a neighborhood $V$ of the diagonal in $X \times X$ and a map $\tau$ from $V \times I$ to $X$ which has the connecting property on $V$. In the first definition $\tau$ is a connecting map for $X$; in the second, $V$ is a connecting neighborhood and $\tau$ is a local connecting map for $X$.

Since every metrizable space can be regarded as a closed subspace of a convex set in a Banach space, it can easily be shown that every AR (ANR)

\footnotetext{
Presented to the Society, August 29, 1963, under the title On equiconnected spaces; received by the editors July $15,1963$.

$\left.{ }^{1}\right)$ This paper was prepared with the support of grant GP-209 from the National Science Foundation. Theorems 4, 5, 7-10 are from the author's Ph.D. dissertation which was completed in June, 1957, at the University of Notre Dame. The author wishes to thank Professor Ky Fan for his assistance in directing that work.
} 
is EC (LEC). It is also easy to show that every LEC space is locally contractible, and that every EC space is both contractible and locally contractible. Hence, for finite-dimensional metrizable spaces, the AR (ANR) and EC (LEC) properties coincide.

It does not seem likely that the EC (LEC) property implies the AR (ANR) property for arbitrary metric spaces. However, in this cornection, the following four theorems have some interest.

THEOREM 1. A topological space $X$ is LEC iff each open covering $\alpha$ of $X$ has an open refinement $\beta$ with the following property: if $Y$ is any space and $f, g$ are $\beta$-near maps from $Y$ to $X$, then there is an $\alpha$-homotopy $h_{t}$ from $Y$ to $X$ such that $h_{t}$ connects $f$ to $g$ and $h_{t}(y)=f(y)$ for all $t$ when $f(y)=g(y)$.

Proof. First suppose that $\tau$ is a local connecting map for $X$ and that $\alpha$ is an open covering of $X$. It is easy to see that there is an open refinement $\beta$ of $\alpha$ such that, for each $U \in \beta, U \times U \times I \subset$ domain $\tau$ and $\tau(U \times U \times I)$ is contained in some member of $\alpha$. Now let $f, g$ be $\beta$-near maps from $Y$ to $X$. Then the homotopy $h_{t}$ defined by $h_{t}(y)=\tau(f(y), g(y), t)$, for $y$ $\in Y, t \in I$, is the desired homotopy.

On the other hand, suppose $X$ satisfies the conditions in the latter half of the theorem. Let $\alpha=\{X\}$ and let $\beta$ be an open refinement as described in the theorem. Let $Y=\bigcup\{U \times U \mid U \in \beta\}$ and define $\beta$-near maps $f$, g from $Y$ to $X$ by $f(x, y)=x, g(x, y)=y$, for $(x, y) \in Y$. Then the resulting $\alpha$-homotopy connecting $f$ to $g$ is a local connecting map for $X$.

We need some terminology before stating the next theorem. Let $V$ be a subset of $X \times X$. A subset $A$ of $X$ is $V$-small iff $A \times A \subset V$. A map $\tau$ from $Y \times I$ to $X$ is $V$-small on $B \subset Y$ iff the set $\tau(y, I)$ is $V$-small for all $y \in B$.

THEOREM 2. If $X$ is a metrizable ANR, then there exists a neighborhood $V$ of the diagonal in $X \times X$ such that for each closed subset $B$ of $V$ and each $V$-small map $\sigma$ from $B \times I$ to $X$ with the connecting property on $B$, there is a local connecting map from $V \times I$ to $X$ which extends $\sigma$.

With $V=X \times X$ and no smallness restriction on $\sigma$, a similar theorem for AR spaces can be obtained. In either case, the converse is unknown.

Proof. This theorem is similar to a theorem proved by $\mathrm{Hu}$ in [9, IV, 1.3]; the argument used here is similar to Hu's. Suppose $X$ is an ANR. Then $X$ is LEC; so let $\lambda$ be a local connecting map for $X$. Let $W$ be a neighborhood of the diagonal $\Delta$ in $X \times X$ such that $(W \circ W) \times I \subset$ domain $\lambda$, and let $V$ be a neighborhood of $\Delta$ such that $V \subset W$ and $\lambda$ is $W$-small on $V$. (This can be done by means of the connecting property of $\lambda$.)

Now let $B$ and $\sigma$ be as described in the statement of the theorem. Let

$$
T=(V \times\{0\}) \cup(B \times I) \cup(\Delta \times I) \cup(V \times\{1\})
$$


and define $\sigma_{*}$ from $T$ to $X$ by

$$
\sigma_{*}(x, y, t)= \begin{cases}x, & \text { if } t=0, \\ \sigma(x, y, t), & \text { if }(x, y) \in B, \\ x, & \text { if }(x, y) \in \Delta, \\ y, & \text { if } t=1 .\end{cases}
$$

$\sigma_{*}$ is clearly well defined and continuous. Since $X$ is ANR and $T$ is closed in $V \times I$, there is a neighborhood $N$ of $T$ in $V \times I$ and an extension $\sigma_{* *}$ from $N$ to $X$ of $\sigma_{*}$. Since $I$ is compact, there is a neighborhood $K$ of $B$ $\cup \Delta$ in $V$ such that $K \times I \subset N . \sigma_{* *}$ has the connecting property on $K$. Moreover, $\sigma_{* *}$ is $V$-small on $B \cup \Delta$, so there is an open subset $L$ of $V$ such that $B \cup \Delta \subset L \subset \bar{L} \subset K$ and $\sigma^{* *}$ is $V$-small on $\bar{L}$.

Let $e$ be a map from $V$ to $I$ such that $e(z)=1$ if $z \in B \cup \Delta$, and $e(z)=0$ if $z \in V-L$. Define $\tau$ on $V \times I$ by

$$
\tau(x, y, t)= \begin{cases}\lambda\left(\lambda(x, y, t), \sigma_{* *}(x, y, t), e(x, y)\right), & \text { if }(x, y) \in \bar{L} \\ \lambda(x, y, t), & \text { if }(x, y) \in V-L .\end{cases}
$$

$\tau$ is the desired local connecting map.

In the following theorem, we obtain a characterization of ANR spaces. In it and in the next theorem, $U^{n+1}$ denotes the topological product of $n+1$ copies of $U$ and $s^{n}$ denotes the Euclidean $n$-simplex with vertices $(1,0, \cdots, 0),(0,1,0, \cdots, 0), \cdots,(0, \cdots, 0,1)$ in Euclidean $(n+1)$-space. A member of $U^{n+1} \times s^{n}$ will be represented by an expression of the form $\left(x_{0}, \cdots, x_{n} ; t_{0}, \cdots, t_{n}\right)$ where $x_{0}, \cdots, x_{n}$ are the coordinates of a point in $U^{n+1}$, and $t_{0}, \cdots, t_{n}$ are the barycentric coordinates of a point in $s^{n}$. The possibility of obtaining this sort of theorem was suggested to the author by Ernest Michael.

Theorem 3. A metrizable space $X$ is an $A N R$ iff there exists an open covering $\alpha$ of $X$ and, for each $n=1,2, \cdots$, a map $\tau_{n}$ from $\bigcup\left\{U^{n+1} \times s^{n} \mid U \in \alpha\right\}$ to $X$ with the following properties:

(a) The map $\tau$ from $\bigcup\left\{U^{2} \times I \mid U \in \alpha\right\}$ to $X$ defined by $\tau\left(x_{0}, x_{1}, t\right)$ $=\tau_{1}\left(x_{0}, x_{1} ; 1-t, t\right)$, if $\left(x_{0}, x_{1}, t\right) \in \bigcup\left\{U^{2} \times I \mid U \in \alpha\right\}$, is a local connecting map for $X$;

(b) For each $n=2,3, \cdots$,

$$
\begin{array}{r}
\tau_{n}\left(x_{0}, \cdots, x_{i-1}, x_{i}, x_{i+1}, \cdots, x_{n} ; t_{0}, \cdots, t_{i-1}, 0, t_{i+1}, \cdots, t_{n}\right) \\
=\tau_{n-1}\left(x_{0}, \cdots, x_{i-1}, x_{i+1}, \cdots, x_{n} ; t_{0}, \cdots, t_{i-1}, t_{i+1}, \cdots, t_{n}\right), \\
\text { if }\left(x_{0}, \cdots, x_{i-1}, x_{i}, x_{i+1} ; t_{0}, \cdots, t_{i-1}, 0, t_{i+1}, \cdots, t_{n}\right) \in \bigcup\left\{U^{n+1} \times s^{n} \mid U \in \alpha\right\} ;
\end{array}
$$

(c) For each neighborhood $V$ of each $x \in X$ there is a neighborhood $W$ of $x$ such that $W^{n+1} \times s^{n} \subset$ domain $\tau_{n}$ and $\tau_{n}\left(W^{n+1} \times s^{n}\right) \subset V$ for all $n=1,2, \cdots$.

REMARK. A similar theorem for AR results if we let $\alpha=\{X\}$, domain $\tau_{n}$ 
$=X^{n+1} \times s^{n}$ for each $n$, and replace "local connecting" in (a) by "connecting."

Proof. Suppose that $X$ is an ANR and that it is a closed subspace of a convex set $Z$ in a Banach space. Since $X$ is an ANR there is a retraction $\rho$ of a neighborhood $K$ of $X$ onto $X$. Let $\beta$ be an open cover of $X$ by convex sets in $Z$ each of which is contained in $K$, and let $\alpha=\{V \cap X \mid V \in \beta\}$. Define $\tau_{n}$ from $\bigcup\left\{U^{n+1} \times s^{n} \mid U \in \alpha\right\}$ to $X$ by

$$
\tau_{n}\left(x_{0}, \cdots, x_{n} ; t_{0}, \cdots, t_{n}\right)=\rho\left(\sum t_{i} x_{i}\right)
$$

if

$$
\left(x_{0}, \cdots, x_{n} ; t_{0}, \cdots, t_{n}\right) \in \bigcup\left\{U^{n+1} \times s^{n} \mid U \in \alpha\right\} .
$$

It is easy to see that, with these definitions of $\alpha$ and $\tau_{n}$, conditions (a) and (b) are satisfied. To prove (c), suppose $V$ is a neighborhood of a point $x$ in $X$. Let $M$ be a convex neighborhood of $x$ such that $M$ is contained in some member of $\beta$ and $\rho(M) \subset V$, and let $W=M \cap X$. Then $W^{n+1} \times s^{n}$ $\subset$ domain $\tau_{n}$ and $\tau_{n}\left(W^{n+1} \times s^{n}\right) \subset \rho(M) \subset V$.

Now suppose there exist $\alpha$ and $\tau_{n}, n=1,2, \cdots$, satisfying (a), (b), and (c). To show that $X$ is an ANR it is sufficient, by a theorem of Hanner $[6$, p. 359], to show that, for each open cover $\beta$ of $X, X$ is $\beta$-dominated by a CW-polytope. So let $\beta$ be a given open cover of $X$. By (c), there exists an open cover $\gamma$ of $X$ which refines $\alpha$ and is such that $\left\{\tau_{n}\left(W^{n+1} \times s^{n}\right) \mid W \in \gamma\right\}$ refines $\beta$ for each $n$. Finally let $\delta$ be a locally finite open cover of $X$ which star-refines $\gamma$.

Let $N$ be the nerve of $\delta$. (For each $U \in \delta$, there is a unique vertex $v_{U}$ of $N$ corresponding to $U . v_{U_{0}}, \cdots, v_{U_{n}}$ are the vertices of a simplex in $N$ iff $\bigcap\left\{U_{i} \mid 0 \leqq i \leqq n\right\} \neq \emptyset$. $N$ has the CW-topology.) Define $\varphi$ from $X$ to $N$ as follows. Let $\left\{\lambda_{U} \mid U \in \delta\right\}$ be a partition of unity on $X$ such that $\lambda_{U}(x)$ $=0$ iff $x \notin U$, for each $U \in \delta$. Then define $\varphi(x)=\sum\left\{\lambda_{U}(x) v_{U} \mid U \in \delta\right\}$ for $x \in X$.

Next we define a map $\psi$ from $N$ to $X$. Let $N_{n}$ be the $n$-dimensional skeleton of $N$ and suppose that $\prec$ is a total ordering of the set $N_{0}$ of vertices of $N$. For each $U \in \delta$ choose $x_{U} \in U$ and define $\psi_{0}$ from $N_{0}$ to $X$ by $\psi_{0}\left(v_{U}\right)$ $=x_{U}$ for each $U \in \delta$. Since $N_{0}$ has the discrete topology, $\psi_{0}$ is continuous. Now supposing that a map $\psi_{n-1}$ from $N_{n-1}$ to $X$ has been defined, define $\psi_{n}$ on $N_{n}$ as follows. If $y \in N_{n-1}$, let $\psi_{n}(y)=\psi_{n-1}(y)$. If $y$ is in an $n$-simplex of $N_{n}$ with vertices $v_{U_{0}}, \cdots, v_{U_{n}}$, written in increasing order, say.

$$
y=\sum \lambda_{i} v_{U_{i}}
$$

let

$$
\psi_{n}(y)=\tau_{n}\left(x_{U_{0}}, \cdots, x_{U_{n}} ; \lambda_{0}, \cdots, \lambda_{n}\right)
$$


Conditions (a) and (b) insure that the maps $\psi_{0}, \psi_{1}, \ldots$ are defined consistently. Define $\psi=\bigcup\left\{\psi_{n} \mid n=0,1, \cdots\right\} . \psi$ is continuous since it is continuous on each closed simplex of $N$.

It remains to show that $\psi \varphi$ is $\beta$-homotopic to the identity map of $X$. Define a homotopy $h$ as follows. If $x \in X$, suppose $U_{0}, \ldots, U_{n}$ are the members of $\delta$ which contain $x$ and that $v_{U_{0}} \prec \cdots \prec v_{U_{n}}$. Then let

$$
h(x, t)=\tau_{n+1}\left(x_{U_{0}}, \cdots, x_{U_{n}}, x ; t \lambda_{U_{0}}(x), \cdots, t \lambda_{U_{n}}(x), 1-t\right) .
$$

Since $\delta$ is a star refinement of $\gamma$ (and hence also of $\alpha$ ), $h$ is well defined. To see that $h$ is continuous, note that each point $x_{0}$ of $X$ has a neighborhood $M$ meeting only finitely many sets, say $U_{0}, \cdots, U_{n}$ of $\delta$. Then, by (b), the formula above defines $h$ for all $x \in M$. Thus $h$ is continuous on $M$. Using (a) and (b), it is clear that $h(x, 0)=x$ and $h(x, 1)=\psi \varphi(x)$ for all $x \in X$. Finally, let $x \in X$, let $U_{0}, \cdots, U_{n}$ be the members of $\delta$ which contain $x$, and let $W \in \gamma$ be such that $\bigcup\left\{U_{i} \mid 0 \leqq i \leqq n\right\} \subset W$. Then $h(x, I)$ $\subset \tau_{n+1}\left(W^{n+2} \times s^{n+1}\right)$, and the latter is contained in some member of $\beta$. So $h$ is a $\beta$-homotopy.

It is natural to look for a condition which a given (local) connecting function for a space can satisfy in order to imply that the space is an AR (ANR). A space $X$ is strictly LEC iff there is a local connecting map $\tau$ for $X$ and, for each $x \in X$, a local base $\beta_{x}$ at $x$ such that, for each $x \in X$ and each $B \in \beta_{x}, \tau$ is defined on $B \times B \times I$ and carries that set into $B$. $X$ is strictly EC iff there is a connecting map for $X$ which makes $X$ at the same time EC and strictly LEC.

THEOREM 4. Every strictly LEC metrizable space is an ANR, and every strictly EC metrizable space is an AR.

Proof. Let $X$ be a strictly LEC metrizable space and let $\tau,\left\{\beta_{x} \mid x \in X\right\}$ be as in the definition above. We shall find an open covering $\alpha$ of $X$ and maps $\tau_{n}, n=1,2, \cdots$, satisfying the conditions of Theorem 3 .

Let $\alpha$ be the set of interiors of members of $\beta=\bigcup\left\{\beta_{x} \mid x \in X\right\}$. Now define $\tau_{n}, n=1, \cdots$, inductively as follows.

$$
\begin{aligned}
& \tau_{1}\left(x_{0}, x_{1} ; t_{0}, t_{1}\right)=\tau\left(x_{0}, x_{1}, t_{1}\right), \quad \text { for }\left(x_{0}, x_{1} ; t_{0}, t_{1}\right) \in \bigcup\left\{U^{2} \times s^{1} \mid U \in \alpha\right\}, \\
& \tau_{n}\left(x_{0}, \cdots, x_{n} ; t_{0}, \cdots, t_{n}\right) \\
& \qquad \begin{cases}\tau\left(\tau_{n-1}\left(x_{0}, \cdots, x_{n-1} ; \frac{t_{0}}{1-t_{n}}, \cdots, \frac{t_{n-1}}{1-t_{n}}\right), x_{n}, t_{n}\right), & \text { if } t_{n} \neq 1, \\
x_{n}, & \text { if } t_{n}=1,\end{cases} \\
& \text { for }\left(x_{0}, \cdots, x_{n} ; t_{0}, \cdots, t_{n}\right) \in \bigcup\left\{U^{n+1} \times s^{n} \mid U \in \alpha\right\}, n>1 .
\end{aligned}
$$

Each $\tau_{n}$ is well defined since, for each $U \in \alpha$, say $U=\operatorname{Int} B$ with $B \in \beta$, and each $n$, we have $\tau_{n}\left(U^{n+1} \times s^{n}\right) \subset B$. Moreover, for each $W$ such that 
$\tau(W \times W \times I) \subset W$ and such that $W \subset U$ for some $U \in \alpha$, we have $\tau_{n}\left(W^{n+1} \times s^{n}\right)=W$ for all $n$; hence (c) in Theorem 3 is satisfied. It is not difficult to show that (a) and (b) are also satisfied. To see that each $\tau_{n}$ is continuous observe that $\tau_{1}$ is continuous, assume that $\tau_{n-1}$ is continuous, and proceed as follows. Let $U \in \alpha$ and define $\pi_{n}: U^{n+1} \times s^{n-1} \times I \rightarrow U^{n+1}$ $\times s^{n}, h_{n}: U^{n+1} \times s^{n-1} \times I \rightarrow X$ by

$$
\begin{aligned}
& \pi_{n}\left(x_{0}, \cdots, x_{n} ; t_{0}, \cdots, t_{n-1} ; t\right)=\left(x_{0}, \cdots, x_{n} ;(1-t) t_{0}, \cdots,(1-t) t_{n-1}, t\right), \\
& h_{n}\left(x_{0}, \cdots, x_{n} ; t_{0}, \cdots, t_{n-1} ; t\right)=\tau\left(\tau_{n-1}\left(x_{0}, \cdots, x_{n} ; t_{0}, \cdots, t_{n-1}\right), x_{n}, t\right),
\end{aligned}
$$

if

$$
\left(x_{0}, \cdots, x_{n} ; t_{0}, \cdots, t_{n-1} ; t\right) \in U^{n+1} \times s^{n-1} \times I .
$$

Then $\pi_{n}$ is a closed map (see [8]) and $h_{n}=\tau_{n} \circ \pi_{n}$. Hence $\tau_{n}$ is continuous iff $h_{n}$ is, and the latter function is clearly continuous.

The second half of the theorem follows since every EC space is contractible and every contractible ANR is an AR.

Unfortunately, the converse of Theorem 4 is not true. The reason is that every point of a strictly LEC space has arbitrarily small closed neighborhoods which are strictly EC, and hence every strictly LEC space is a local AR. If we let AR(C) denote the class of compact metric AR's, it follows that every compact metric strictly LEC space is a local $\operatorname{AR}(C)$. But there is an example due to Borsuk [2] of a space which is an AR(C) but not a local AR(C).

In the remainder of the paper we prove some theorems on the union of EC and LEC spaces analogous to the well-known theorems of Hanner [7], and Aronszajn and Borsuk, [1] and [3], for AR's and ANR's.

3. Local Properties of LEC. It is easy to see that every open subset $U$ of an LEC space $X$ is also an LEC space. In fact if $\tau$ is a local connecting map for $X$, then such a map can be found for $U$ by restricting $\tau$ to a suitable neighborhood of the diagonal in $U \times U$. We also have the following.

Theorem 5. Let $X_{1}$ and $X_{2}$ be two open sets in a metrizable space $X$ whose union is $X$. Then $X$ is LEC iff $X_{1}$ and $X_{2}$ are LEC. Moreover, if $\tau_{1}, \tau_{2}, \tau$ are local connecting maps for $X_{1}, X_{2}, X$, respectively, we may require, for $i=1,2$, that $\tau_{i}$ be defined on domain $\tau-X_{j} \times X_{j} \times I, j \neq i$, and agree with $\tau$ there.

Proof. Let $d$ be a metric for $X$ (in this and in all the remaining theorems). Also use $d$ to denote the distance between a point and a set. Let

$$
\begin{array}{ll}
A_{i}=\left\{x \in X \mid d\left(x, X_{i}-X_{j}\right)<2 d\left(x, X_{j}-X_{i}\right)\right\}, & i \neq j, \\
B_{i}=\left\{x \in X \mid d\left(x, A_{i}-A_{j}\right)<2 d\left(x, A_{j}-A_{i}\right)\right\}, & i \neq j, \\
A=A_{1} \cap A_{2} . &
\end{array}
$$


Then $A_{1}, A_{2}, B_{1}, B_{2}$ are open, $X=A_{1} \cup A_{2}=B_{1} \cup B_{2}$, and $\bar{B}_{i} \subset A_{i} \subset \overline{A_{i}}$ $\subset X_{i}, i=1,2$. Moreover, if $\tau_{1}, \tau_{2}$ are local connecting maps for $X_{1}, X_{2}$, then there is an open neighborhood $V$ of the diagonal in $X \times X$ such that

$$
\begin{gathered}
V \subset\left(B_{1} \times B_{1}\right) \cup\left(B_{2} \times B_{2}\right), \\
\left(V \cap\left(A_{i} \times A_{i}\right)\right) \times I \subset \operatorname{domain} \tau_{i}, \quad i=1,2,
\end{gathered}
$$

and

$$
\left(\tau_{1}(x, y, t), \tau_{2}(x, y, t), u\right) \in \operatorname{domain} \tau_{1}
$$

if

$$
(x, y) \in V \cap(A \times A), \quad t \in I, u \in I .
$$

We shall define a local connecting map on $V$. First define $\varphi$ from $V$ to $I$ by $\varphi(x, y)=\min \{1, \psi(x, y)\}$, for $(x, y) \in V$, where

$$
\psi(x, y)=\frac{d\left(x, B_{1}-B_{2}\right) d\left(y, B_{1}-B_{2}\right)}{d\left(x, B_{2}-B_{1}\right) d\left(y, B_{2}-B_{1}\right)} .
$$

The fraction $\psi(x, y)$ is never indeterminant; for example, if $(x, y) \in V$ and $x \in B_{i}-B_{j}$, then $y \in B_{i}$ and $d\left(x, B_{j}-B_{i}\right) d\left(y, B_{j}-B_{i}\right)>0$. If $\psi(x, y)$ is of the form $a / 0$, with $a \neq 0$, then $\psi(x, y)=+\infty$ and $\varphi(x, y)=1$. It is necessary to check the continuity of $\varphi$ at a point $\left(x_{0}, y_{0}\right)$ of $V$ only if either $x_{0}$ or $y_{0}$ is not in $B_{1}$. So suppose $x_{0} \notin B_{1}$. Then $x_{0} \in B_{2}-B_{1}$ and $y_{0} \in B_{2}$. Choose $\epsilon>0$ such that the $\epsilon$-neighborhoods about $x_{0}$ and $y_{0}$ do not meet the closed set $B_{1}-B_{2}$. Then if $d\left(x, x_{0}\right)<\epsilon, d\left(y, y_{0}\right)<\epsilon$, we have

$$
\psi(x, y)>\frac{\left(d\left(x_{0}, B_{1}-B_{2}\right)-\epsilon\right)\left(d\left(y_{0}, B_{1}-B_{2}\right)-\epsilon\right)}{\epsilon\left(d\left(y_{0}, B_{2}-B_{1}\right)+\epsilon\right)}
$$

and, if $\epsilon$ is small enough, then $\psi(x, y)>1$ and $\varphi(x, y)=1$.

Now define $\tau$ from $V \times I$ to $X$ by

$$
\tau(x, y, t)= \begin{cases}\tau_{1}(x, y, t), & \text { if }(x, y) \in V-\bar{B}_{2} \times \bar{B}_{2}=V_{1}, \\ \tau_{2}(x, y, t), & \text { if }(x, y) \in V-\bar{B}_{1} \times \bar{B}_{1}=V_{2}, \\ \tau_{1}\left(\tau_{1}(x, y, t), \tau_{2}(x, y, t), \varphi(x, y)\right), & \text { if }(x, y) \in V \cap(A \times A)=V_{3} .\end{cases}
$$

$\tau$ obviously has the connecting property if it is a map. To see that $\tau$ is a map, observe that $V=V_{1} \cup V_{2} \cup V_{3}$ and that $V_{1}$ and $V_{2}$ are disjoint. Next suppose that $(x, y) \in V_{i} \cap V_{3}$. If $i=1, \varphi(x, y)=0$; if $i=2, \varphi(x, y)=1$. In either case the definition of $\tau$ is consistent and, of course, all three of the sets $V_{1}, V_{2}, V_{3}$ are open.

The last assertion in the theorem follows from the definition of $\tau$ and the fact that $\bar{B}_{i} \subset X_{i}, i=1,2$.

Since the pairwise disjoint union of open LEC's is clearly LEC, it follows that LEC is a G-hereditary property (see Michael [11, p. 166]) for metric 
spaces. I.e., if $X$ is a metric space and $Y$ any subspace, then

(a) If $Y$ is LEC then every open subspace of $Y$ is LEC.

(b) If $Y$ is the union of two open LEC's, then $Y$ is LEC.

(c) If $Y$ is the union of a pairwise disjoint collection of open LEC spaces, then $Y$ is LEC.

By Theorem 3.6 of [11], a paracompact space $X$ has a given topological property $\mathscr{P}$ if $X$ has property $\mathscr{P}$ locally and $\mathscr{P}$ is G-hereditary for $X$. So, if a local LEC space is one in which each point has an LEC neighborhood, then

Theorem 6. A metrizable space is LEC iff it is a local LEC.

4. Unions of Closed EC's and LEC's. In this section we prove theorems for unions of closed EC's and LEC's similar to those of Aronszajn and Borsuk [1] for AR's and ANR's.

Lemma 1. Let a metrizable space $X$ be the union of two closed sets $A$ and $B$, let $V_{A}$ be an $X \times X$ neighborhood of the diagonal of $A \times A$, let $V_{B}$ be a $B \times B$ neighborhood of the diagonal of $B \times B$, and let $\tau_{A}, \tau_{B}$ be maps from $V_{A} \times I, V_{B} \times I$, respectively, to $X$ such that

(a) $\tau_{B}\left(V_{B} \times I\right) \subset B$ and $\tau_{B}$ is a local connecting map for $B$;

(b) $\tau_{A}$ has the connecting property on $(A \times A) \cap V_{A}$;

(c) $\tau_{A}(x, y, 0)=x, \tau_{A}(x, y, 1)=y$, if $(x, y) \in V_{A}$;

(d) $\tau_{A}(x, y, I) \subset B$, if $(x, y) \in V_{A} \cap((X-A) \times(X-A))$.

Then $X$ is LEC and there is a local connecting map $\tau$ for $X$ such that $\tau_{A}$ is defined at each point of $(A \times A \times I) \cap$ domain $\tau$ and agrees with $\tau$ there.

Proof. Define a map $u$ from $(X-A) \times(X-A) \times I$ to $I$ by

$$
u(x, y, t)= \begin{cases}\min \left\{1, \frac{t d(x, y)}{d(x, A) d(y, A)}\right\}, & \text { if } 0 \leqq t \leqq \frac{1}{2}, \\ \min \left\{1, \frac{(1-t) d(x, y)}{d(x, A) d(y, A)}\right\}, & \text { if } \frac{1}{2} \leqq t \leqq 1 .\end{cases}
$$

Observe that $u(x, y, 0)=u(x, y, 1)=u(x, x, I)=0$ for all $x, y \in X-A$.

For each $a \in A$ there is an open neighborhood $W_{a}$ of $a$ in $X$ such that $\tau_{A}$ is defined everywhere on $W_{a} \times W_{a} \times I, \tau_{B}$ is defined everywhere on $\left(W_{a} \cap B\right) \times\left(W_{a} \cap B\right) \times I, \quad$ and $\quad\left(\tau_{B}(x, y, t), \tau_{A}(x, y, t)\right) \in V_{B}, \quad$ if $(x, y, t)$ $\in\left(W_{a}-A\right) \times\left(W_{a}-A\right) \times I$. Then $W=\bigcup\left\{W_{a} \mid a \in A\right\}$ is an open neighborhood of $A$ and $V=\bigcup\left\{W_{a} \times W_{a} \mid a \in A\right\}$ is an open neighborhood of the diagonal of $W \times W$. Define $\lambda$ from $V \times I$ to $X$ by

$$
\lambda(x, y, t)= \begin{cases}\tau_{B}\left(\tau_{B}(x, y, t), \tau_{A}(x, y, t), u(x, y, t)\right), \\ \quad \text { if }(x, y) \in V \cap((X-A) \times(X-A)), \\ \tau_{A}(x, y, t), & \text { otherwise. }\end{cases}
$$


The precautions taken before its definition insure that $\lambda$ is a function. $\lambda$ has the connecting property on $V$ and agrees with $\tau_{A}$ on $(A \times A \times I)$ $\cap(V \times I)$. It is clear that $\lambda$ is continuous at $\left(x_{0}, y_{0}, t_{0}\right) \in V \times I$ if $\left(x_{0}, y_{0}\right)$ $\in(X-A) \times(X-A)$ or if $\left(x_{0}, y_{0}\right)$ is in the interior of $(X \times A) \cup(A \times X)$. It remains to prove continuity when $\left(x_{0}, y_{0}\right) \in \operatorname{Bd}((X-A) \times(X-A))$. So suppose $x_{0} \in \operatorname{Bd}(X-A)$ and $y_{0} \in \overline{X-A}$. Then $\lambda\left(x_{0}, y_{0}, t\right)=\tau_{A}\left(x_{0}, y_{0}, t\right)$ for all $t \in I$.

Let $\epsilon>0$ and, in the following, suppose

$$
(x, y) \in V \cap((X-A) \times(X-A)) .
$$

To prove that $\lambda$ is continuous at $\left(x_{0}, y_{0}, t_{0}\right)$ it is sufficient to show, for $(x, y)$ close enough to $\left(x_{0}, y_{0}\right)$, that $d\left(\lambda(x, y, t), \lambda\left(x_{0}, y_{0}, t\right)\right)<\epsilon$ for all $t$.

If $x_{0}=y_{0}$, the argument is trivial, since in that case

$$
\lambda\left(x_{0}, x_{0}, I\right)=\tau_{A}\left(x_{0}, x_{0}, I\right)=\tau_{B}\left(x_{0}, x_{0}, I\right)=x_{0} .
$$

So suppose $x_{0} \neq y_{0}$. By using the connecting property of $\tau_{B}$ and (c), it is easily seen that there exist $\delta_{0}>0, t_{0}>0$ such that $d\left(\lambda(x, y, t), \lambda\left(x_{0}, y_{0}, t\right)\right)$ $<\epsilon$ whenever $d\left(x, x_{0}\right)<\delta_{0}, d\left(y, y_{0}\right)<\delta_{0}$, and either $t<t_{0}$ or $t>1-t_{0}$. Next pick $0<\delta_{1}<\delta_{0}$ such that

$$
\frac{d(x, y)}{d(x, A) d(y, A)}>\frac{d\left(x_{0}, y_{0}\right)-2 \delta_{1}}{\delta_{1}\left(\delta_{1}+d\left(y_{0}, A\right)\right)} \geqq \frac{1}{t_{0}},
$$

whenever $d\left(x, x_{0}\right)<\delta_{1}$ and $d\left(y, y_{0}\right)<\delta_{1}$. Finally choose $0<\delta<\delta_{1}$, such that $d\left(\tau_{A}(x, y, t), \tau_{A}\left(x_{0}, y_{0}, t\right)\right)<\epsilon$, for all $t \in I$, whenever $d\left(x, x_{0}\right)<\delta$ and $d\left(y, y_{0}\right)<\delta$.

Now suppose $d\left(x, x_{0}\right)<\delta, d\left(y, y_{0}\right)<\delta$. By the choice of $\delta_{1}$, we have $u(x, y, t)=1$ and $\lambda(x, y, t)=\tau_{A}(x, y, t)$ whenever $t_{0} \leqq t \leqq 1-t_{0}$. Hence, by the fact that $\lambda\left(x_{0}, y_{0}, t\right)=\tau_{A}\left(x_{0}, y_{0}, t\right)$ and by the choices of $\delta_{0}$ and $\delta$, we have $d\left(\lambda(x, y, t), \lambda\left(x_{0}, y_{0}, t\right)\right)<\epsilon$ for all $t$. This completes the proof that $\lambda$ is continuous.

Since $W$ is open and $\lambda$ has the connecting property on the neighborhood $V$ of the diagonal in $W \times W$, there is a smaller neighborhood $V_{W}$ of the diagonal in $W \times W$ such that $\lambda\left(V_{W} \times I\right) \subset W$. Hence $W$ is an LEC with local connecting map $\tau_{W}=\lambda \mid V_{W} \times I$. Now observe that $X-A$ is an open subspace of the LEC space $B$, so that $X$ is the union of the two open LEC spaces $W$ and $X-A$. By Theorem $5, X$ is LEC and there is a local connecting map $\tau$ for $X$ that agrees with $\tau_{W}$ on domain $\tau-(X-A) \times(X-A)$ $\times I$ and, in particular, with $\tau_{A}$ on $(A \times A \times I) \cap$ domain $\tau$.

Theorem 7. A neighborhood retract $A$ of a metrizable LEC space $X$ is also an LEC space. Moreover, if $\lambda$ is a local connecting map for $A$, then there is a local connecting map $\tau$ for $X$ such that $\lambda$ is defined at each point of $(A \times A \times I)$ $\cap$ domain $\tau$ and agrees with $\tau$ there. 
Proof. The first assertion is obvious. To prove the second, suppose that the diameter of $X$ is less than $1 / 4$, that $\tau_{X}$ is a local connecting map for $X$ with domain $V_{X} \times I$, and that $\rho$ is a retraction of a neighborhood of $A$ onto A. Define $\varphi(x, y)=d(x, A)+d(y, A)$ for $(x, y) \in X \times X$. $\varphi$ is continuous and $0 \leqq \varphi<1-\varphi \leqq 1$.

We will apply Lemma 1 with $B=X, \tau_{B}=\tau_{X}$. So we need an $X \times X$ neighborhood $V_{A}$ of the diagonal in $A \times A$ and a map $\tau_{A}$ defined on $V_{A}$ $X I$, satisfying (b), (c), and (d) of Lemma 1 . For each $a \in A$, there is an open neighborhood $U_{a}$ of $a$ such that $\left(U_{a} \cap A\right) \times\left(U_{a} \cap A\right) \times I \subset$ domain $\lambda$, $U_{a} \subset$ domain $\rho, U_{a} \times \rho\left(U_{a}\right)$ and $\rho\left(U_{a}\right) \times U_{a}$ are contained in $V_{X}$, and $\rho\left(U_{a}\right)$ $\times \rho\left(U_{a}\right) \times I$ is contained in domain $\lambda$. Then $V_{A}=\bigcup\left\{U_{a} \times U_{a} \mid a \in A\right\}$ is an $X \times X$ neighborhood of the diagonal of $A \times A$. Define $\tau_{A}$ on $V_{A} \times I$ by $\tau_{A}(x, y, t)=\lambda(x, y, t)$, if $(x, y) \in V_{A} \cap(A \times A)$, and otherwise by

$$
\tau_{A}(x, y, t)= \begin{cases}\tau_{X}(x, \rho(x), t / \varphi), & \text { if } 0 \leqq t \leqq \varphi, \\ \lambda(\rho(x), \rho(y),(t-\varphi) /(1-2 \varphi)), & \text { if } \varphi \leqq t \leqq 1-\varphi, \\ \tau_{X}(\rho(y), y,(t-1+\varphi) / \varphi), & \text { if } 1-\varphi \leqq t \leqq 1,\end{cases}
$$

where $\varphi=\varphi(x, y)$.

$\tau_{A}$ is clearly continuous at $\left(x_{0}, y_{0}, t_{0}\right) \in V_{A} \times I$ if $\left(x_{0}, y_{0}\right)$ is in the interior of $A \times A$ or in the complement of $A \times A$. The proof of continuity when $\left(x_{0}, y_{0}\right) \in \operatorname{Bd}(A \times A)$ is tedious but routine. The essential facts needed are that $(t-\varphi(x, y)) /(1-2 \varphi(x, y))-t$ tends to 0 uniformly for $t \in I$ as $(x, y)$ tends to $\left(x_{0}, y_{0}\right)$, and that given $\epsilon>0$ there is a $\delta>0$ such that $d\left(\lambda(x, y, u), \lambda\left(x_{0}, y_{0}, t\right)\right)<\epsilon$ whenever $d\left(x, x_{0}\right)<\delta, d\left(y, y_{0}\right)<\delta$, and $|u-t|<\delta$. However, we omit the details.

Since $V_{A}, V_{X}, \tau_{A}$, and $\tau_{X}$ satisfy the hypotheses of Lemma 1 , with $X=B$, there is a local connecting map $\tau$ for $X$ such that $\tau_{A}$ (and hence $\lambda$ ) is defined at each point of $(A \times A \times I) \cap$ domain $\tau$ and agrees with $\tau$ there.

Theorem 8. Let $A, B$ be two closed sets in a metrizable space $X$ whose union is $X$, and suppose that there is a retraction $\rho$ of a neighborhood of $A$ $\cap B$ in $X$ onto $A \cap B$. Then $A$ and $B$ are LEC iff $X$ is LEC.

Proof. The "if" part is trivial since $A$ and $B$ are neighborhood retracts of $X$. So suppose that $A$ and $B$ are LEC. Applying Theorem 7 twice, we obtain local connecting maps $\lambda_{A}, \tau_{B}$, for $A, B$ respectively, which agree on the common part of their domains. Let domain $\lambda_{A}=W_{A} \times I$, domain $\tau_{B}$ $=V_{B} \times I$. Without loss of generality we may assume that $W_{A}=\left(W_{A} \cup V_{B}\right)$ $\cap(A \times A), V_{B}=\left(W_{A} \cup V_{B}\right) \cap(B \times B)$. Thus the function $\tau=\lambda_{A} \cup \tau_{B}$ is continuous and has the connecting property on $W_{A} \cup V_{B}$. But this set is not a neighborhood of the diagonal in $X \times X$. So proceed as follows.

Let $r$ be a retraction of a neighborhood of $A$ onto $A$ which agrees with $\rho$ on $B \cap$ domain $r$. (In particular, $r$ leaves points of $B$ in $A \cap B$.) Then 
for each $a \in A$ we can choose an open neighborhood $U_{a}$ of $a$ small enough that the sets

$$
\left(U_{a} \cap A\right) \times\left(U_{a} \cap A\right),\left(U_{a} \cap B\right) \times r\left(U_{a} \cap B\right), r\left(U_{a} \cap B\right) \times\left(U_{a} \cap B\right),
$$

and $r\left(U_{a}\right) \times r\left(U_{a}\right)$ are all contained in $W_{A} \cup V_{B} . V_{A}=\bigcup\left\{U_{a} \times U_{a} \mid a \in A\right\}$ is an $X \times X$ neighborhood of the diagonal of $A \times A$. Define $\tau_{A}(x, y, t)$ for $(x, y, t) \in V_{A} \times I$ by

$$
\tau_{A}(x, y, t)= \begin{cases}\tau(x, r(x), 3 t), & \text { if } 0 \leqq t \leqq 1 / 3 \\ \tau(r(x), r(y), 3 t-1), & \text { if } 1 / 3 \leqq t \leqq 2 / 3, \\ \tau(r(y), y, 3 t-2), & \text { if } 2 / 3 \leqq t \leqq 1\end{cases}
$$

It is easy to see that $V_{A}, V_{B}, \tau_{A}, \tau_{B}$ satisfy the conditions of Lemma 1 , and hence that $X$ is an LEC.

Using simpler but analogous arguments one can also obtain the following two theorems.

Theorem 9. A retract $A$ of a metrizable EC space is also an EC space. Moreover, if $\lambda$ is a connecting map for $A$, then there is a connecting map for $X$ which agrees with $\lambda$ on $A \times A \times I$.

Theorem 10. Let $A, B$ be two closed sets in a metrizable space $X$ such that $X=A \cup B$ and $A \cap B$ is a retract of $X$. Then $A$ and $B$ are $E C$ iff $X$ is $E C$.

\section{REFERENCES}

1. N. Aronszajn and K. Borsuk, Sur la somme et le produit combinatoire des rétractes absolus, Fund. Math. 18 (1932), 193-197.

2. K. Borsuk, On an irreducible 2-dimensional absolute retract, Fund. Math. 37 (1950), 137-160.

3. __ Über eine Klasse von lokal zusammenhängenden Räumen, Fund. Math. 19 (1932), 220-242.

4. J. Dugundji, Absolute retracts and local connectedness in arbitrary metric spaces, Compositio Math. 13 (1955), 229-246.

5. R. Fox, On fibre spaces. II, Bull. Amer. Math. Soc. 49 (1943), 733-735.

6. O. Hanner, Retraction and extension of mappings of metric and non-metric spaces, Ark. Mat. 2 (1952), 315-360.

7. __ Some theorems on absolute neighborhood retracts, Ark. Mat. 1 (1951), 389-408.

8. C. J. Himmelberg, On the product of quotient spaces, Amer. Math. Monthly (to appear).

9. S. T. Hu, Theory of retracts, Wayne State Univ. Press, Detroit, Mich. (to appear).

10. C. Kuratowski, Topologie. II, Monogr. Mat., Warsaw, 1950.

11. E. Michael, Local properties of topological spaces, Duke Math. J. 21 (1954), 163-171.

12. J. -P. Serre, Homologie singulière des espaces fibrés, Ann. of Math. 54 (1951), 425-505.

UNIVERSITY OF KANSAS,

LAWRENCE, KANSAS 